\title{
Fourier analysis of movement patterns in pigeons
}

\author{
FRANCISCO J. SILVA \\ Indiana University, Bloomington, Indiana \\ and \\ JOSEPH J. PEAR, ROBERT W. TAIT, and JAMES J. FOREST \\ University of Manitoba, Winnipeg, Manitoba, Canada
}

\begin{abstract}
Fourier analysis describes the amount of variation in a time series by partitioning it into sinusoids of different amplitudes and wavelengths and quantifying the extent to which these waves are synchronized. The importance of a given wave is represented by its contribution to the explained variance of the time series. To illustrate its potential application to the study of movement, Fourier analysis was used to quantify the movement patterns of 2 pigeons on a variable-interval (VI) and a variable-time (VT) schedule of reinforcement. For comparison, the movements of 2 pigeons that did not receive reinforcement were similarly analyzed. Characteristic frequencies were higher during VI than during VT, and relatively low during nonreinforcement. We concluded that Fourier analysis can be used to represent movement patterns compactly, study their periodicity, stereotypy, and resonance, and assist in computer simulations and models of movement.
\end{abstract}

Increasingly, behavioral researchers are using advances in computer technology to study the real-time position of a subject as it moves in three-dimensional (3-D) space. Techniques have been reported for monitoring the movements of fish (see, e.g., Kirkpatrick, Schneider, \& Pavlovski, 1991; Scherer, McNicol, \& Capel, 1992), rodents (e.g., Clarke, Smith, \& Justesen, 1992; Livesey \& Leppard, 1981; Renner, Pierre, \& Schilcher, 1990), and humans (e.g., Atkeson \& Hollerbach, 1985; see also Jouen \& Lepecq, 1989; Woltring, 1984). Examples of behavioral research areas in which continuous spatial tracking techniques are used include the dynamics of hand and arm movements involved in sign language (e.g., Jennings \& Poizner, 1988; Poizner, Wooten, \& Salot, 1986) and movement patterns of pigeons exposed to various reinforcement contingencies (e.g., Eldridge, Pear, Torgrud, \& Evers, 1988; Pear \& Legris, 1987; Silva, Silva, \& Pear, 1992).

An issue confronting researchers who collect movement data is how best to characterize it. Typically, the ultimate representation requires a great deal of selectivity, and only brief segments of at most a few sessions are reported. One feature of the data that is often not explored quantitatively is its periodicity. Given that the regularities (i.e., periodicities) in the rate of discrete responses is a focus of study in at least one important subdivision of psychology (see, e.g., Ferster \& Skinner, 1957), a quantitative examination of the periodicity of movement patterns might be an important step in relating individual responses to the larger movement of which they are a part. Quantifying such a re-

\footnotetext{
We thank B. Timberlake, E. Hearst, and K. Silva for their comments on earlier drafts of this manuscript. Correspondence should be addressed to F. Silva, Department of Psychology, Indiana University, Bloomington, IN 47405 (e-mail: fsilva@indiana.edu).
}

lationship might, for example, provide valuable insights regarding the formation and dynamics of behavioral units. In addition, the periodicity of movement patterns may constitute a rich source of data for refuting or extending the generalizations drawn from research on discrete responses. In a related point concerning the tendency of operant behaviorists to use discrete responses to index learning, Angermeier (1984) commented that to obtain a general formulation "the entirety of the learning process must be subjected to an analysis which seeks to determine its regularities and irregularities" (p. 141, emphasis in the original).

The present article reports an application of Fourier analysis to extract and summarize the periodicity in continuous steady-state movement data collected under several reinforcement contingencies. Although other techniques for examining periodicity exist (e.g., average waveform, Houpt, 1990; demodulation, Sing, Thorne, Hegge, \& Babkoff, 1985 ), there were logical and pragmatic reasons for choosing Fourier analysis in the present application. First, a large amount of theoretical work regarding the assumptions and mathematical techniques of Fourier analysis has been done, so its assumptions and constraints are well understood (Fox, 1989; Fox \& Hayes, 1985; Press, Flannery, Teukolsky, \& Vetterling, 1989). Second, Fourier analysis has proven fruitful in summarizing both the cyclic and "noisy" nature of the phenomena of other sciences (e.g., the motion of a pendulum, sun spot activity, and fluctuations in the economy). Third, Fourier analysis is related to contemporary mathematical analyses and theories (e.g., fractal geometry, chaotic systems, Fox, 1989) that are becoming important in psychology (DeAngelis, 1993; see, e.g., Abraham, Abraham, \& Shaw, 1990; Barton, 1994; Butz, 1992; Hoyert, 1992; Killeen, 1989; Schmid, 1991). Finally, there is the pragmatic consideration that a variety of Fourier analysis programs are available for personal and mainframe computers. 
Although Fourier analysis has been used to examine the periodicity of discrete responses (e.g., Broadbent, 1994), the technique can also be used to examine movement patterns (especially if those patterns exhibit periodicity). The present study concentrated on the continuous movement patterns generated by pigeons exposed to a variable-interval (VI) schedule, in which reinforcement is contingent on the first keypeck after intervals varying around a constant mean, and to a variable-time (VT) schedule, in which reinforcement occurs independently of responding after intervals varying around a constant mean. Typically, pigeons exposed to VI schedules engage in steady rates of keypecking (Ferster \& Skinner, 1957) and stereotyped movements such as pacing and walking in looping patterns (see Pear, 1985; Pear, Silva, \& Kincaid, 1989). The stable keypeck responding generated by VI schedules makes them useful as behavioral barometers for studying the effects of various independent variables and for evaluating new analytical techniques (e.g., Pear, 1985; Pear et al., 1989). VT schedules, less frequently studied than VI schedules, are used to study the effects of response-independent reinforcement. Typically, VT schedules generate stereotyped movement patterns such as pacing (Buzzard \& Hake, 1984; Matthews, Bordi, \& Depollo, 1990; Staddon \& Simmelhag, 1971). Examinations of movement patterns during response-dependent and response-independent reinforcement have identified schedule-induced differences in how pigeons distribute locomotor activity between reinforcements (e.g., Eldridge et al., 1988; Timberlake \& Lucas, 1985). If Fourier analysis is a sensitive analytical procedure, it should also differentiate the movement patterns exhibited with the two schedules.

\section{FOURIER ANALYSIS}

Periodic processes can be analyzed by a class of computational methods that falls under the general rubric of Fourier transform methods or spectral analyses. ${ }^{1}$ Fourier techniques are applicable to the analysis of time series whose means and variances are independent of time and whose means and autocorrelation values are equivalent when calculated from different samples of the same length within the series. Many physical processes, including the behavior of an animal, approximate these requirements (Sturgis, 1983).

With a Fast Fourier Transform (FFT), it is possible to describe the amount of variation in a time series by partitioning it into sinusoids of different amplitudes and frequencies, and then quantifying the extent to which these waves are synchronized. A sinusoid is a wave whose variance can be accounted for by a single frequency, or, as Kaplan (1983) defined it, a sinusoid is a projection produced by a point moving around a circle at a constant rate. The diameter of the circle is the peak-to-peak amplitude of the sinusoid, and a sinusoid's frequency is the number of complete passages around the circumference of the circle per unit of time traveled by the moving point.
Knowledge of its amplitude and frequency, however, is insufficient to describe a sinusoid completely. It is also important to know where on the circle the point started its motion. A point that started its motion from the extreme right ( 3 o'clock) on the circle would be $180^{\circ}$ out of phase with a sinusoid of the same amplitude and frequency that started its motion from the extreme left ( 9 o'clock). Thus, to describe a sinusoid, it is necessary to specify the wave's amplitude, frequency, and phase (represented by a deviation from an arbitrary position on the hypothetical circle with the deviation measured by the sine and cosine of the angle subtended at the center of the circle by the starting position of the point and the arbitrary position).

An FFT fits a set of sinusoids with different amplitudes, frequencies, and phases to a time series. The degree of fit between a particular sinusoid and the time series is reflected in its contribution to the explained variance of the series, just as the importance of a linear contrast in an analysis of variance (ANOVA) is judged by its contribution to the total variance. Also, just as a set of contrasts applied to data in an ANOVA must be orthogonal, any set of sinusoids must be orthogonal in order to carry out appropriate statistical tests of those sinusoids. For a sinusoid of a given frequency, only one other sinusoid at that same frequency is orthogonal to it-the sine perspective is orthogonal to the cosine perspective.

The variance of a time series is measured by dividing it into orthogonal sinusoids and measuring the deviations of those sinusoids from the mean of the series. The variance of the sinusoids sum to the variance of the original series, making the fraction of the time series' variance at a given frequency analogous to the proportion of variance accounted for by a pair of contrasts (except for some scale factors that depend on the physical system). A given frequency is analogous to a pair of contrasts because the variance at any frequency may be associated with two components in sine and cosine perspectives. Moreover, because the two components are orthogonal, their variances are additive. Thus, a time series can be partitioned into a sum of sine and cosine sinusoids in which each orthogonal pair of sinusoids exhausts the variance at one frequency. The same is done for each frequency up to the Nyquist limit, which is the maximum number of cyclic components that can be determined for a given sample length. The Nyquist frequency corresponds to one half the sampling rate. For example, if the sampling rate is 600 times per minute, then, in a 1 -min period, it is possible to assess the amount of variance accounted for by frequencies from 1 through 300 cycles per minute.

A standard way of representing the amount of variance accounted for by each frequency is to construct a frequency spectrum (also called a periodogram, deterministic spectrum, or amplitude spectrum) or, if the variances are squared, a power spectrum, where the abscissa is frequency and the ordinate is "power." In addition, it is possible to construct a phase spectrum, which shows the starting point of each sinusoid relative to a conventionally designated origin 
(usually the extreme right of a circle, or 0 in the sine perspective, moving counterclockwise). Phase or phase shift is normally expressed as an angle between $-180^{\circ}$ and $+180^{\circ}$ or, equivalently, between $-\pi$ and $+\pi$ radians. For example, consider two sinusoids with identical amplitudes and frequencies, but in which the phase shift of one wave is one-half $\pi$ (i.e., $1.57 \mathrm{rad}$, or $90^{\circ}$ ) and the phase shift of the other is $\pi$ (i.e., $3.14 \mathrm{rad}$, or $180^{\circ}$ ). Two such sinusoids differ only to the extent that the origin of one wave is onequarter cycle ahead of the other wave. For an idealized (and highly improbable) behavioral example, imagine a pigeon walking at a constant rate in a counterclockwise circle while simultaneously bobbing its head in an exact pendulum motion toward a response key in such a manner that it pecks the key on each pass by it. In this example, if we measured the projection of the distance of the bird's head from the response key, then the circular movement pattern and the pendulum-like pecking pattern would each produce a sinusoid. Assuming that the origin of the bird's movement coincided with the initiation of a keypeck, the two sinusoids would have different amplitudes but identical frequencies and phases. Because the circling and pecking originated at the "top of the circle," the sinusoids produced by these behaviors would be shifted one-half $-\pi$ (i.e., $-1.57 \mathrm{rad}$, or $-90^{\circ}$ ) with respect to sine.

In generating a spectrum from a time series, three constraints of the FFT need to be considered. First, the interpretation of the spectrum is affected by the length and starting point of the data series to be transformed. If a sample length is selected that spans two or more distinct behavioral processes, the resulting spectrum would provide the average composition of the two processes and consequently would accurately represent neither (Fox \& Hayes, 1985; Kaplan, 1983). Second, the computational requirements of the FFT require that the number of data points used to calculate a spectrum be an integer power of 2 (i.e., sample lengths of $2,4,8,16,32$, etc., data points). Third, for a given sample length, the maximum number of cyclic components that can be determined (Nyquist limit) is equal to one half the sampling rate. Thus, the resolution of the spectrum is determined by the number of data points in the series, to the nearest integer power of 2 , and the rate at which they were sampled.

Additionally, to produce more easily interpretable spectra, two mathematical transformations are commonly performed. One transformation is to convert the frequency spectrum to a power spectrum by squaring the amplitudes at each frequency in the analysis to accentuate the more important frequencies. The other is a trigonometric transformation performed on the original time series to minimize the effect of fitting multiple frequencies to the same set of data points. An example will clarify the basis for this transformation. Consider a time series whose variation is mostly described by a sinusoid with a frequency of 30 cycles/ $\mathrm{min}$. The same time series would also be well described by sinusoids with frequencies of 29 and 31 cycles $/ \mathrm{min}$. Although a sinusoid with a frequency of $30 \mathrm{cycles} / \mathrm{min}$ accounts for the largest portion of the variance, the power at- tributed to this frequency may be underestimated because of the apparent contributions of adjacent frequencies to the power spectra. To correct and improve the estimates of dominant frequencies, trigonometric transformations are used to produce an estimate of the best fitting sinusoid and to reduce the identification of spurious power to adjacent frequencies (see Press et al., 1989, pp. 466-472, for a discussion of the relative strengths and weaknesses of the available transformations).

\section{METHOD}

Four pigeons served in this experiment. Burds 1 and 2 were exposed first to several sessions of VI 5-min and then to several sessions of VT 5-min, and Birds 3 and 4 were not given any programmed reinforcement contingencies. Tracking was done by a unit termed a video-acquisition module that analyzed the signals from two orthogonally oriented black-and-white video cameras. One horizontal coordinate $(x)$ was computed on one camera, and the other horizontal and the vertical coordinates $(y, z)$ were computed on the other. Discrimination between the target and the background was made on the basis of relative brightness. The cameras were electronically linked so that they scanned the observed scene synchronously at $30 \mathrm{~Hz}$. The video signal from each camera was analyzed line by line from the top of the image, until, when the minimum width and darkness criteria were satisfied, the highest and darkest target was identified. Because the pigeon's head was darkened with black shoe polish, it was the highest dark object in the otherwise white experımental environment. It should be noted, however, that the same point on the bird was not always the point being tracked. For example, if the bird tilts its head to one side, the highest point might shift from the top center of the head to a point more toward the side of the head. The resolution of the system was $\pm 0.25 \mathrm{~cm}$. The maximum error was $\pm 0.75 \mathrm{~cm}$.

Logic circuits within the video-acquisition module determined the horizontal and vertical positions of the target relative to each camera and converted the positions to Cartesian coordinates relative to the location of the pecking key. The data were averaged in blocks of three, yieldıng 10 data points/sec (for additional details, see Pear, Rector, \& Legris, 1982, or Pear \& Eldridge, 1984).

The Fourier analyses were done using a Macintosh Plus. The data were parsed into (1) time and (2) distance of the pigeon's head from the response key. The Fourier analyses of the changes over time in distance of the bird's head from the key were conducted using a commercially available statistical analysis program (Systat, 1987).

As noted, it is important to consider the constraints of the FFT before generating a spectrum from a time series. First, we had to select the length and starting point of the sample series to be transformed so that the sample did not span two or more distinct behavioral processes. To achieve this we excluded movements during the occurrence of a reinforcer, which abruptly changes the trajectory of the movement pattern by requiring the bird to approach the feeder. We also excluded data during the first $300 \mathrm{sec}$ of the session from the Fourier analyses because they contained warm-up effects that obscured regularities in the subsequent spectra. Second, consideration of the computational requirements of the FFT required that the number of data points used to calculate a spectrum was an integer power of 2. On the basis of these considerations, $51.2 \mathrm{sec}$ of data (i.e., 512 data points) were used to calculate each spectrum. In the experimental session, spectra were sampled at $200-\mathrm{sec}$ increments beginning at $300 \mathrm{sec}$ after the start of the session and ending with the interval at $1,700 \mathrm{sec}$ into the session.

To improve the estimates of dominant frequencies, all data were smoothed with a Hanning function prior to the Fourier analysis (see Press et al., 1989). The resulting amplitudes were squared, generating power spectra. The data for these analyses were taken from the 
last session of each phase for Birds 1 and 2 , and from the first session for Birds 3 and 4 because the latter two birds exhibited little movement following their first session.

\section{ILLUSTRATIONS OF DISCRETE RESPONDING, MOVEMENT PATTERNS, AND FOURIER ANALYSIS}

Figure 1 shows data from the last session of VI 5-min and VT 5-min for Birds 1 and 2 in the format typically used in our previous reports of behavioral experiments using continuous tracking (e.g., Eldridge et al., 1988; Silva et al., 1992). At the top are cumulative keypecking records taken from the entire session. During VI 5-min, both birds engaged in fairly steady rates of keypecking, as indicated by the linear form of their cumulative records. During VT 5-min, both birds' rate of keypecking decreased substantially, as indicated by the more shallow-sloped cumulative records.

The figure panels directly below the cumulative records show the distance of the bird's head from the response key over a 51-sec period in the middle of the session (900$951 \mathrm{sec}$ ). These plots show that both birds made repetitive excursions from the back of the chamber to the key throughout the last sessions of VI 5-min and VT 5-min; however, the birds did not move as close to the key during VT 5-min as they did during VI 5-min.

The plots next to the distance versus time plots show data from the same time sample plotted in the $x y$ plane. Bird 1's movement pattern during this $51-\mathrm{sec}$ portion of

BIRD 1
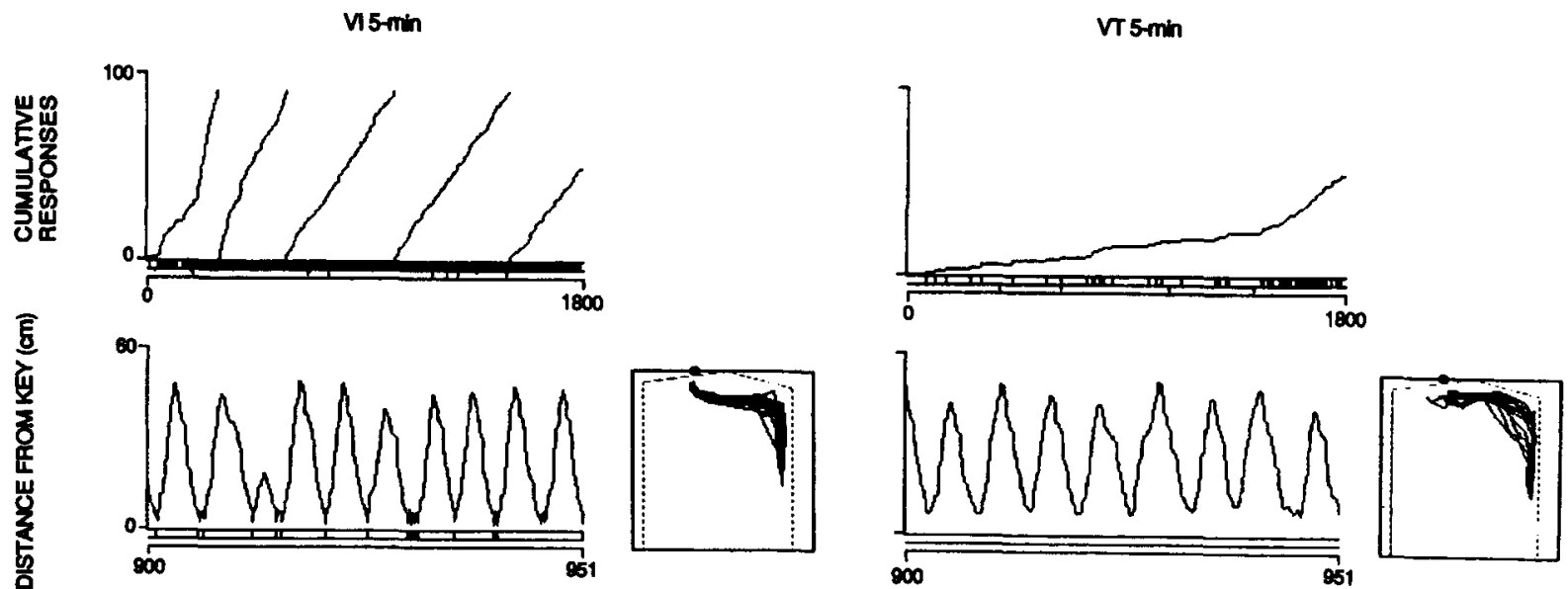

BIRD 2
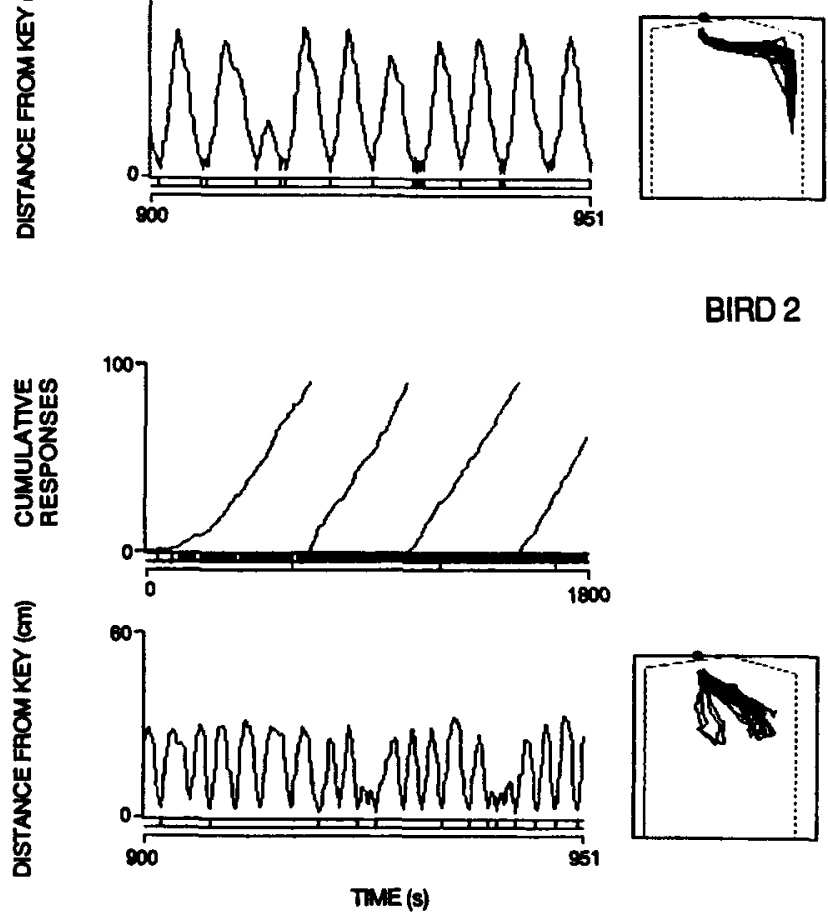

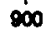
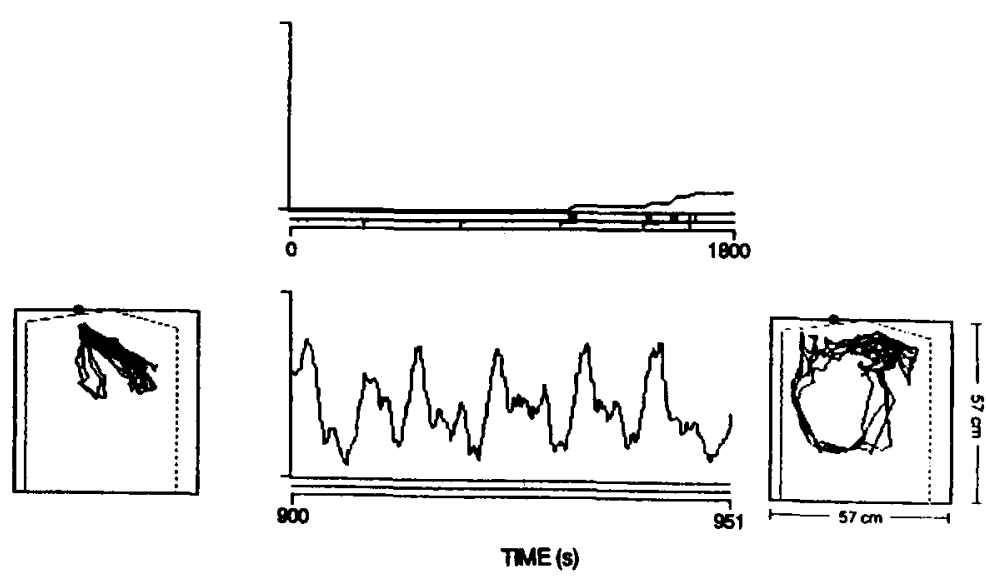

Figure 1. Last session of VI 5-min and VT 5-min for Birds 1 and 2. The top graphs for each bird are cumulative keypecking records taken from the entire session. The horizontal band just below each record shows keypecks as vertical marks, which, because of their density, sometimes form a solid black strip. The horizontal band below the keypeck band shows instances of reinforcement. The graphs below the cumulative records show the distance of the bird's head from the response key for 51 sec in approximately the middle of the session (900-951 sec). The bird's distance from the key was determined trigonometrically by using the raw $x$-, $y$-, and $z$-coordinates of the bird's and the response key's locations. As with the cumulative records, the horizontal band just below each graph shows keypecks as vertical marks. The plots next to the distance versus time plots show data from the same time sample plotted in the $x y$ plane (top view). The position of the response key is indicated by the black dot at the top of each panel. The dashed lines indicate regions of the chamber from which data could not be obtained because of the orientation of the cameras. 
VI 5-min consisted of walking away from the vicinity of the key along the right side of the feeder wall, walking along the right wall toward the rear of the chamber, and then turning around and walking along the right wall back toward the key. Bird 1's movement pattern was similar during the corresponding 51-sec portion of VT 5-min, except that the bird did not move as close to the key during VT 5-min as it did during VI 5-min. Bird 2's movement pattern from 900 to $951 \mathrm{sec}$ during VI 5-min consisted of walking in an elongated clockwise loop approximately 20 times at an angle of about $45^{\circ}$ to the front wall, followed by walking in a single, more pronounced counterclockwise loop almost perpendicular to the front wall. Bird 2 walked in alternating clockwise (the small, dense circles to the right of the key) and counterclockwise loops (the large circles), however, during a corresponding 51 -sec portion of VT 5-min.

The top row in Figure 2 contains plots of the birds' distance-from-key data displayed as power spectra plotted across time, which permits an examination of changes in the characteristic frequencies of the movement patterns within a session. Each plot was constructed from eight 51.2 -sec samples starting at $300 \mathrm{sec}$ and taken every $200 \mathrm{sec}$ starting from the first keypeck after the start of the time sample. The second row in Figure 2 shows contour plots of the data in the top row. These plots contain the same information as the power spectra in the top row, although the information is presented differently. Each line within the frame is a contour line and denotes an increase of 20 units of amplitude squared (i.e., variability accounted for). The closer the contour lines, the steeper the gradient. The interpretation of the contours is isomorphic to the 3-D power spectra in the top row.

During VI 5-min, Bird 1's dominant movement occurred approximately 13 times $/ \mathrm{min}$. As the session progressed, there was a tighter distribution across the frequency domain, with the maximum shifting to a slightly higher frequency. Bird 1's tightly packed contour lines during the VI 5-min data segment shown indicate that a narrow range of spatial frequencies near 13 cycles/min describes the bird's behavior and that the peak frequency shifted upward as the session progressed. During VT 5-min, Bird 1's dominant movement was slower than during VI 5-min, occurring approximately 9 times/min. However, early in the session (time $=500 \mathrm{sec}$ ) this bird engaged in movements that occurred approximately 3 times $/ \mathrm{min}$.

Bird 2's spectral distributions during VI 5-min were flatter and broader than those for Bird 1. Bird 2's dominant movement occurred approximately 16 to 18 times/min at the beginning and end of the session. Bird 2 also engaged in movements that occurred approximately 3 times $/ \mathrm{min}$. Comparing Figure 2 with Figure 1 suggests that this frequency corresponded to counterclockwise looping. Bird 2's contour lines are far apart, forming a shallow gradient and indicating that a broader spectrum of frequencies is required to describe the movements of this bird. This contour plot also shows that the $18 \mathrm{cycle} / \mathrm{min}$ frequency and the 16 cycle/min frequency account for a greater proportion of variability early and late, respectively, in the session.
Like that of Bird 1, Bird 2's behavior during VT 5-min was characterized by a different spectral distribution. Bird 2's dominant movement during VT 5-min contained two significant components, one that occurred approximately 7 times/min and another that occurred approximately 13 times $/ \mathrm{min}$. Bird 2's contour lines through most of the VT 5-min data segment shown indicate that a narrower range of frequencies describes the bird's behavior. The $7 \mathrm{cycles} / \mathrm{min}$ frequency and the $13 \mathrm{cycles} / \mathrm{min}$ frequency accounted for most of the variability.

The third row in Figure 2 shows plots of the birds' data displayed as phase spectra. Note that there does not appear to be any simple relationship between phase, frequency, and time during a session for either bird during VI 5-min or VT 5-min. The contour plots of the phase spectra shown in the fourth row appear also to show a mathematically complex relationship.

Plots of the data for Birds 3 and 4, which were not trained to keypeck, are shown in Figure 3. The top and bottom rows in the figure are plots of Bird 3 and 4's data displayed as power spectra across time and their corresponding contour plots, and phase spectra across time and their corresponding contour plots. Because these birds did not receive any programmed schedule of reinforcement, they controlled for effects other than those of the VI and VT schedules to which Birds 1 and 2 were exposed.

No single frequency accounts for Bird 3's movements, although the power spectra show that the bird occasionally engaged in regular movements occurring approximately 3 times/min. Bird 4's power spectrum shows that its movements occurred at the same rate as Bird 3's, except that low-frequency movements accounted for more of the variability. On the following session (which is not shown), the power spectra for both birds were almost entirely flat at zero (indicating almost no movement). As with Birds 1 and 2 , the phase spectra show no readily recognizable relationship between phase, frequency, and time during a session.

\section{CONCLUSIONS AND APPLICATIONS}

The data presented above demonstrate that Fourier analysis can meaningfully summarize the periodicity in the movement patterns that occur on at least two basic schedules of reinforcement, namely, VI and VT. The fact that Fourier analysis compacted the data while quantifying and highlighting the periodicity in those data provides much to recommend it in studies of movement patterns (e.g., Pear, 1985; Scherer et al., 1992; see also Silva \& Pear, 1995). It is also interesting to note that discrete measures of behavior - such as rate of keypecking - give, in comparison with plots of the birds' movements and Fourier analysis, a rather inaccurate impression of behavior by indicating that VT, for example, resulted in keypeck levels close to zero during much of the sessions shown in Figure 1. However, the Fourier analyses of the movement patterns show that periodic behavior occurred at above-zero frequencies even after many sessions of VT training. Finally, the present results confirm previous observations that VT schedules control locomotor behavior in the ab- 
BIRD 1

VI 5-min

VT 5-min

\section{POWER SPECTRA}
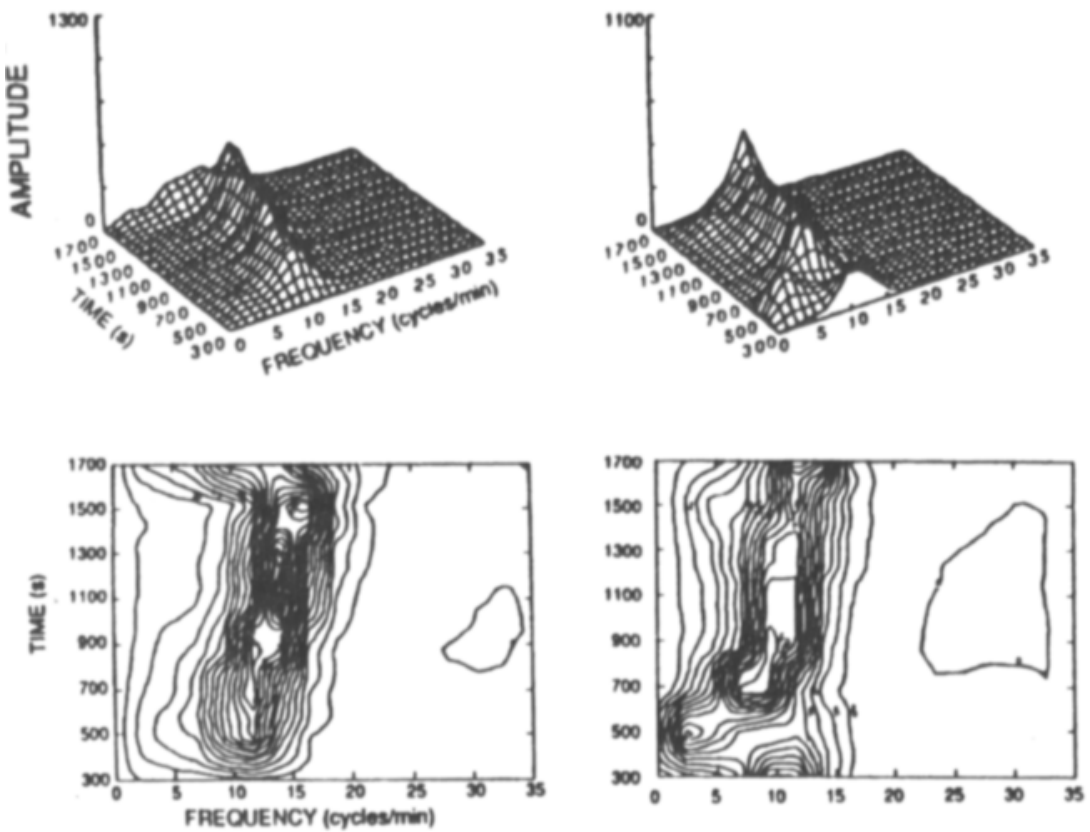

PHASE SPECTRA
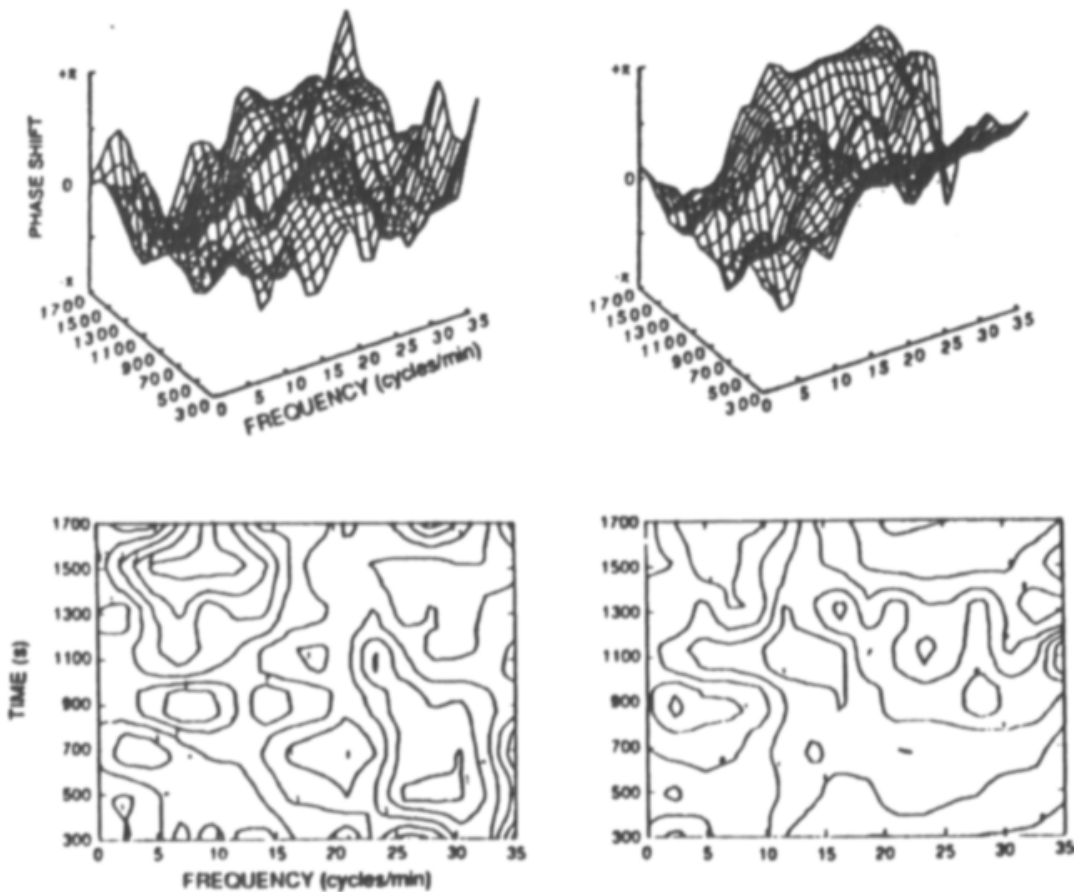

Figure 2. Power and phase spectra from the last session of VI 5-min and VT 5-min for Birds 1 (above) and 2 (next page). The top row shows plots of the birds' data displayed as power spectra plotted across time. Each plot was constructed from eight, 51.2-sec samples starting at 300 sec and taken every 200 sec starting from the first keypeck after the start of the time sample. The second row shows contour representations of the data in the top row. These plots contain the same information as the power spectra in the top row. Each line within the frame is a 


\section{BIRD 2}

VI 5-min

VT 5-min

\section{POWER SPECTRA}
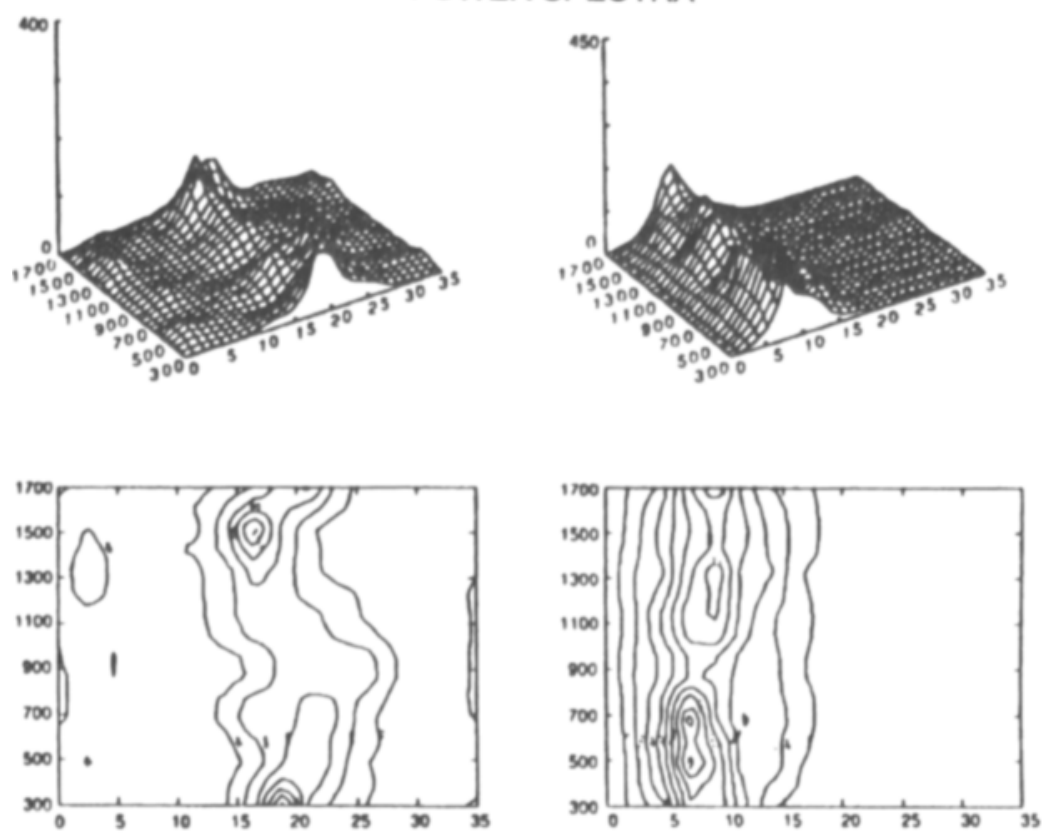

PHASE SPECTRA
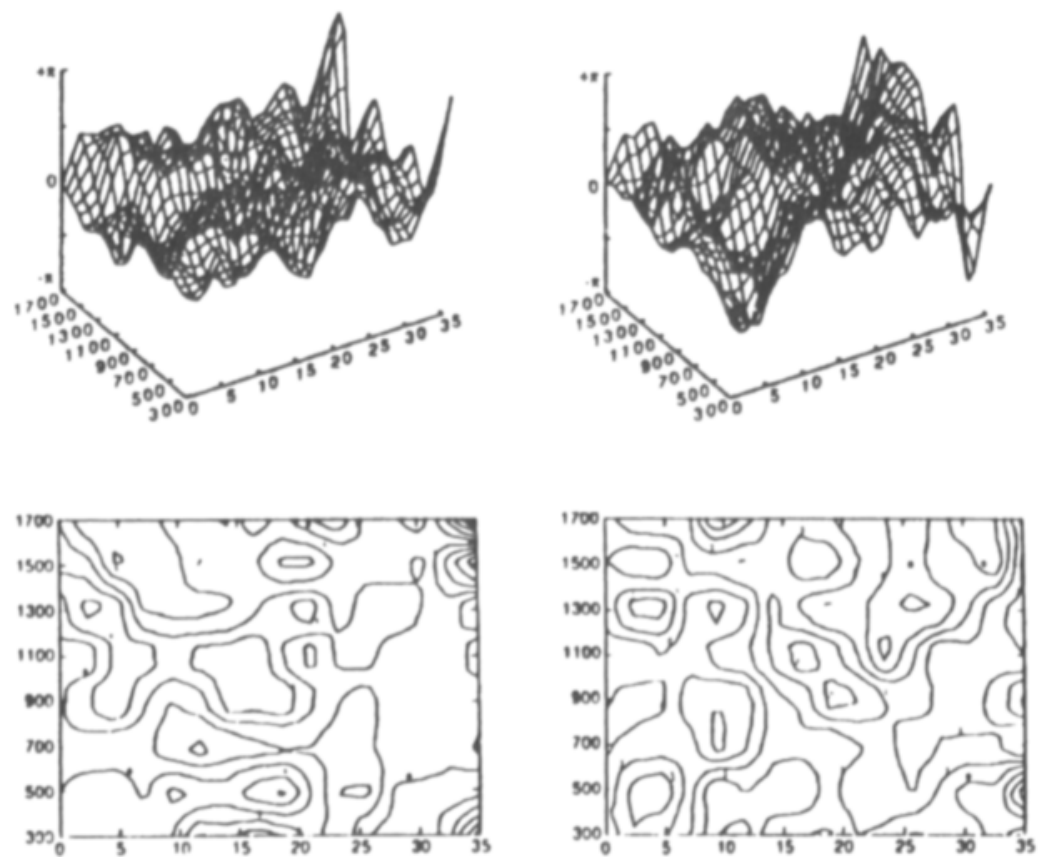

contour line and denotes an increase of 20 units of amplitude squared (i.e., variability accounted for). The closer the contour lines are together, the steeper the gradient. The interpretation of the contours is isomorphic with the power spectra in the top row. The third and fourth rows show plots of the birds' data displayed as phase spectra and as contour plots of the phase spectra. Each line within the contour plot denotes a shift of 0.2 radians. 


\section{POWER SPECTRA}

BIRD 3
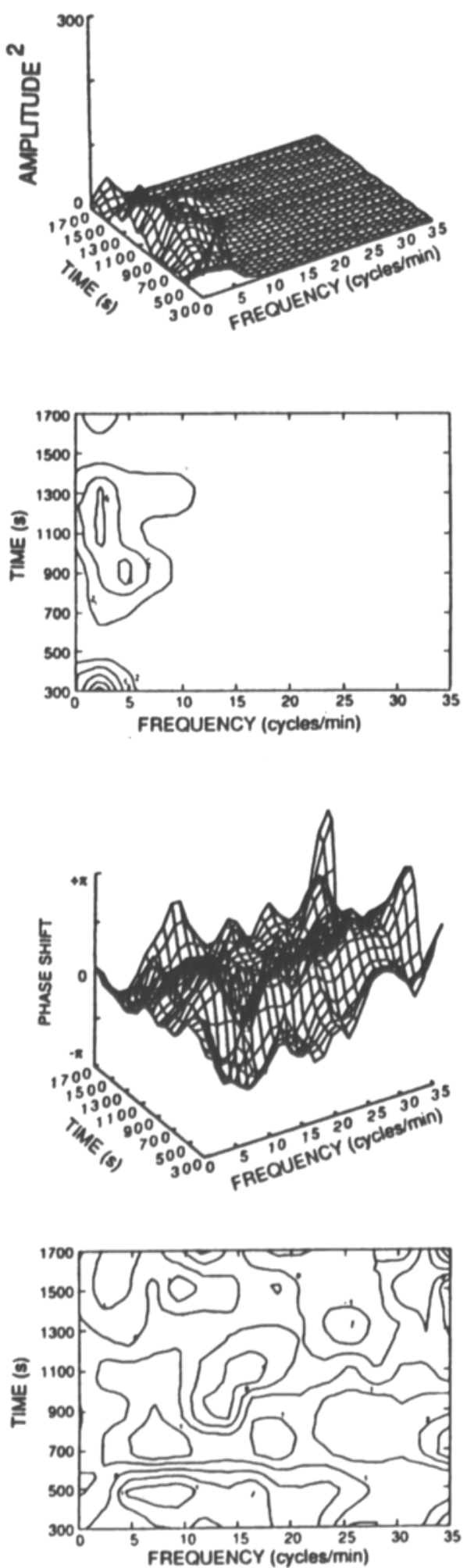

BIRD 4
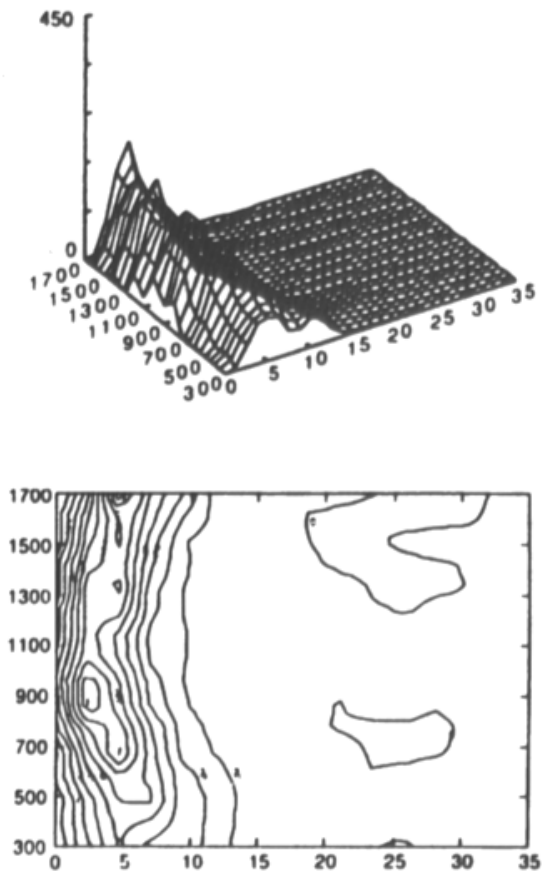

PHASE SPECTRA

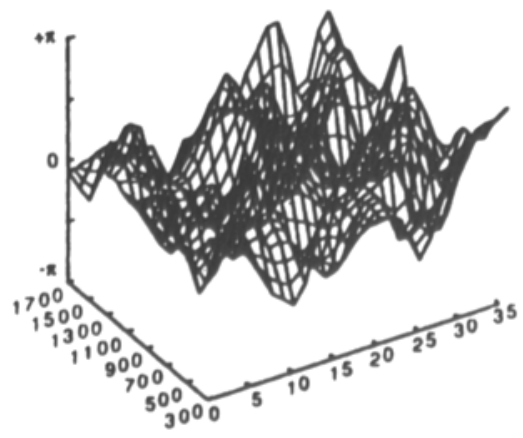

Figure 3. Power and phase spectra from the first experimental session for Birds 3 and 4. Details are the same as those for Figure 2. 
sence of a terminal discrete response such as keypecking (Matthews et al., 1990; Staddon \& Simmelhag, 1971; Timberlake \& Lucas, 1985).

Although it is well established that a discrete response previously contingent on reinforcement decreases during response-independent reinforcement (e.g., Eldridge et al., 1988; Lachter, 1971; Timberlake \& Lucas, 1985), the effect of such a change in the reinforcement contingency on the frequency of the movement patterns was unknown. During VT, decrements in the response previously required for reinforcement are thought to occur because reinforcement no longer consistently and reliably follows this response. As a result, other behaviors - some that may be incompatible with the previously reinforced response (e.g., moving away from the response key)-are occasionally reinforced. Similarly, one might expect that movement patterns associated with the performance of the discrete response would "drift" in their form for a similar reason during response-independent reinforcement. However, there is no a priori reason to assume that the frequencies characterizing movement patterns should decrease rather than increase or remain unchanged when reinforcement is changed from a response-dependent to a responseindependent schedule. This effect, together with the fact that movement patterns during VI and VT were characterized by higher frequencies than during nonreinforcement (Birds 3 and 4), suggests that pigeons may have a bias toward low-frequency movements. The effect of responsedependent reinforcement may be to accelerate the movements of hungry birds to a higher frequency that maximizes reinforcement. Moreover, if pigeons have a bias toward lowfrequency movements, then the decrement in the previously reinforced response during response-independent reinforcement may be related to the expression of this bias rather than solely being the outcome of other behavior being reinforced (see Lachter, 1971).

In sum, Fourier analysis was sensitive to differences in movement patterns supported by VI and VT schedules, as well as during reinforcement (Birds 1 and 2) and nonreinforcement (Birds 3 and 4). Higher frequencies appear to characterize movement patterns during VI compared with those during VT. Higher frequencies also appear to characterize movements during reinforcement compared with those during nonreinforcement. These two outcomes suggest that pigeons may have a bias toward low-frequency movements. The effect of response-dependent reinforcement may be to accelerate the movements of hungry birds to a higher frequency that maximizes reinforcement. This constellation of effects suggests that Fourier analysis is sensitive to changes in movement patterns, and the interpretation of these effects provides novel insights, hypotheses, and points of departure for further studies of movement.

One property that makes Fourier analysis a powerful analytical tool is its ability to express a time series (e.g., distance from the response key) in the frequency domain (i.e., power spectra) both exactly and completely. This correspondence between the time series and the frequency domain allows detailed analyses of the spectra with the assurance that there is an exact analog in the time series (Fox \& Hayes, 1985). To maintain this correspondence, it is necessary to retain both the amplitude and the phase components of the Fourier transformed series. However, despite describing exactly half the information of the movement patterns and defining the origin in space of all component sinusoids, the phase spectra in this study provided little insight into the structure of the movement patterns. It appears that the relationship between phase, frequency, and time during a session is mathematically complex. Nevertheless, the phase components (along with the amplitude components) are necessary for a reconstruction of the original time series.

It is important to note that we are not suggesting that Fourier analysis should always be included in an analysis of movement patterns. Clearly, there are many instances when the form and direction of the movement is of critical interest (e.g., Jennings \& Poizner, 1988; Silva et al., 1992; see also data depicted in Figure 1). What we are suggesting is that researchers monitoring movement patterns and interested in behavioral periodicity consider using Fourier analysis. In short, it is a powerful analytic tool that might usefully be incorporated with other analytic methods. Moreover, its use and focus on periodicity might enhance the study of phenomena related to movement such as stereotypy and resonance (e.g., Silva \& Pear, 1995), as well as aiding in computer simulations and models of movement patterns. For example, through the use of Fourier analysis, the stereotypy of a movement pattern could be defined as the minimum number (or range) of frequencies necessary to account for $80 \%$ of the variance in a time series. Thus, if six frequencies (or a range of six frequencies) account for $80 \%$ of the variance in a subject's movements, and four frequencies (or a range of four frequencies) do the same for another subject's movements, we would conclude that the latter subject's movements are more stereotyped. In this manner, stereotypy can be measured on a continuous dimension in which the amount of stereotypy is determined by the number of frequencies necessary to account for $n \%$ of the variance in the time series.

Similarly, Fourier analysis can also be used to study the resonance of movement patterns. Resonance is a physical property of objects that vibrate more readily at one frequency than at another. This property is easily demonstrated in the domain of physical systems. Hineline (1986) argued that a property analogous to physical resonance is applicable to cyclic animal movement (fundamentally, a "vibration bias"), in that varying the frequency of an inducing event, such as the frequency of feedback or reinforcement, should alter the frequency of the movement pattern. This hypothesis is testable using Fourier analysis because it implies that different (fixed) frequencies of feedback should produce movements that are characterized by different power spectra. Thus, the dominant spectral frequency should be quantitatively related to the frequency of feedback (cf. Killeen, 1975). This use of Fourier analysis to study 
response biases has a precedent in work showing that spatial biases of hummingbirds can be meaningfully characterized and studied using Fourier analysis (e.g., Cahoon, 1987).

In the present application, Fourier analysis of movement patterns showed that when Birds 1 and 2 were shifted from response-dependent reinforcement to responseindependent reinforcement, the dominant movement changed to a lower frequency. As noted, pigeons might have a bias toward low-frequency movements, and the effect of response-dependent reinforcement may be to accelerate the movements of hungry birds to a higher frequency that maximizes reinforcement. Note that Birds 3 and 4 , which were not trained on reinforcement schedules, displayed a trend toward low-frequency movements.

In relation to computer simulations of movement patterns, Fourier analysis could provide valuable quantitative data about the periodicity of the behavior. An experimenter might, for example, have a particular set of theoretical parameters and equations that could be modeled in a computer program (e.g., Beer, Ritzman, \& McKenna, 1993), and the resulting hypothetical movement patterns could be subjected to Fourier analysis. Comparison of such results with data from behavioral studies could potentially lead to a more sophisticated understanding of behavioral units and dynamics, and provide a strong empirical basis for theories that characterize the continuous nature of behavior (e.g., Killeen, 1994; see also Pear, 1994).

Conducting inverse FFTs on power and phase spectra reproduce the raw data used to generate those power and phase spectra. By altering spectral output and performing inverse FFTs on behavioral data, it might be possible to gain insights into the relationship between spectra and a variety of movement patterns. The complexity of these simulations could be enhanced by adding numerous parameters to the simulation such as level of deprivation, rate of feedback, experimental conditions, and organismic biases. Additional sophistication could be introduced by relating Fourier coefficients to simple linear regression and fractal dimension, both of which are mathematically related to Fourier analysis (Fox, 1989; Fox \& Hayes, 1985; Mandelbrot, 1982). Mathematical work involving fractal dimension may in turn lead to an examination of movement patterns as self-similar phenomena.

\section{REFERENCES}

Abraham, F. D., Abraham, R. H., \& Shaw, C. D. (1990). A visual introduction to dynamical systems theory for psychology. Santa Cruz, CA: Aerial Press.

ANGERMEIER, W. F. (1984). The evolution of operant learning and memory. New York: Karger.

AtKeson, C. G., \& Hollerbach, J. M. (1985). Kinematic features of unrestrained vertical arm movements. Journal of Neuroscience, $\mathbf{5}$, 2318-2330.

BARTON, S. (1994). Chaos, self-organization, and psychology. American Psychologist, 49, 5-14.

Beer, R. D., Ritzman, R. E., \& MCKenna, T. (EDs.) (1993). Biological neural networks in invertebrate neuroethology and robotics. Boston: Academic Press.

BLOOMFIELD, P. (1976). Fourier analysis of time series: An introduction. New York: Wiley.
Broadbent, H. A. (1994). Periodic behavior in a random environment. Journal of Experimental Psychology: Animal Behavior Processes, 20, 156-175.

Brockwell, P. J., \& Davis, R. A. (1987). Time series: Theory and methods. New York: Springer-Verlag.

Butz, M. R. (1992). The fractal nature of the development of the self. Psychological Reports, 71, 1043-1063.

BuzzaRD, J. H., \& HAKE, D. F. (1984). Stimulus control of scheduleinduced activity in pigeons during multiple schedules. Journal of the Experimental Analysis of Behavior, 42, 191-209.

CAHOON, P. (1987). Detecting and eliminating spatial bias when tracking foraging behavior in a laboratory experiment. In M. L. Commons, A. Kacelnik, \& S. J. Shettleworth (Eds.), Quantitative analyses of behavior: Foraging (Vol. 6, pp. 255-274). Hillsdale, NJ: Erlbaum.

Clarke, R. L., Smith, R. F., \& Justesen, D. R. (1992). A programmable proximity-contact sensor to detect location or locomotion of animals. Behavior Research Methods, Instruments, \& Computers, 24, 515-518.

DeANGELIS, T. (1993). Chaos, chaos everywhere is what the theorists think. APA Monitor, 24, 1, 41 .

Eldridge, G. D., Pear, J. J., Torgrud, L. J., \& Evers, B. H. (1988). Effects of prior response-contıngent reinforcement on superstitious behavior. Animal Learning \& Behavior, 16, 277-284.

FERSTER, C. B., \& SKINNER, B. F. (1957). Schedules of reinforcement. New York: Appleton-Century-Crofts.

Fox, C. G. (1989). Empincally derıved relatıonships between fractal dimension and power law form frequency spectra. In C. H. Scholz \& B. B. Mandelbrot (Eds.), Fractals in geophysics (pp. 211-239). Berlin: Birkhauser Verlag.

Fox, C. G., \& HAYES, D. E. (1985). Quantitative methods for analyzing the roughness of the seafloor. Reviews of Geophysics, 23, 1-48.

HiNELINE, P. N. (1986). Re-tuning the operant-respondent distınction. In T. Thompson \& M. D. Zeiler (Eds.), Analysis and integration of behavioral units (pp. 55-79). Hillsdale, NJ: Erlbaum.

Houpt, T. A. (1990). Circadia: An integrated circadian data analysis program for the Apple Macintosh. Cambridge, MA: Behavioral Cybernetics.

HOYERT, M. S. (1992). Order and chaos in fixed-interval schedules of reinforcement. Journal of the Experimental Analysis of Behavior, $\mathbf{5 7}$, 339-363.

Jennings, P. J., \& Poizner, H. (1988). Computer graphic modelling and analysis II Three-dimensional reconstruction and interactive analysis. Journal of Neuroscience Methods, 24, 45-55.

JOUEN, F., \& LEPECQ, J-C. (1989). Optical and electronic systems for spatial and temporal analysis of video images. Behavior Research Methods, Instruments, \& Computers, 21, 2-10.

KAPLAN, H. L. (1983). Correlations, contrasts, and components: Fourier analysis in a more familiar termınology. Behavior Research Methods \& Instrumentation, 15, 228-241.

KILleEN, P. [R.] (1975). On the temporal control of behavior. Psychological Review, 82, 89-115.

KILLEEN, P. R. (1989). Behavior as a trajectory through a field of attractors. In J. R. Brink \& C. R. Haden (Eds.), The computer and the brain. Perspectives on human and artificial intelligence (pp. 53-82). Amsterdam: Elsevier, North-Holland.

KILLEEN, P. R. (1994). Mathematical principles of reinforcement based on the correlation of behavior with incentives in short-term memory. Behavioral \& Brain Sciences, 17, 105-172.

Kirkpatrick, T., Schneider, C. W., \& PAvloski, R. (1991). A computerized infrared monitor for following movement in aquatic animals. Behavior Research Methods, Instruments, \& Computers, 23, 16-22.

LACHTER, G. D. (1971). Some temporal parameters of non-contingent reinforcement. Journal of the Experimental Analysis of Behavior, 16, 207-217.

LIVESEY, P. J., \& LEPPARD, K. (1981). A TV monitored system for recording open-field activity in the rat. Behavior Research Methods \& Instrumentation, 13, 331-333.

MANDELBROT, B. B. (1982). The fractal geometry of nature. San Francisco: Freeman.

Matthews, T. J., BoRd, F., \& Depollo, D. (1990). Schedule-induced kınesis and taxic behavioral stereotypy in the pigeon. Journal of Experimental Psychology: Animal Behavior Processes, 16, 335-344.

PEAR, J. J. (1985). Spatiotemporal patterns of behavior produced by 
variable-interval schedules of reinforcement. Journal of the Experimental Analysis of Behavior, 44, 217-231.

PEAR, J. J. (1994). Problems and pitfalls for Killeen's principles of reinforcement. Behavioral \& Brain Sciences, 17, 146-147.

Pear, J. J., \& EldRIDGE, G. D. (1984). The operant-respondent distinction. Journal of the Experimental Analysis of Behavior, 42, 453-467.

PEAR, J. J., \& Legris, J. A. (1987). Shaping by automated tracking of an arbitrary operant response. Journal of the Experimental Analysis of Behavior, 47, 241-247.

PeAR, J. J., Rector, B. L., \& Legris, J. L. (1982). Toward analyzing the continuity of behavior. In M. L. Commons, R J. Herrnstein, \& H. Rachlin (Eds.), Quantitative analyses of behavior Vol. 2 Matching and maximizing accounts (pp. 3-24). Cambridge, MA: Ballinger.

Pear, J. J., Silva, F. J., \& Kincaid, K. M. (1989). Three-dimensional spatiotemporal imaging of movement patterns: Another step toward analyzing the continuity of behavior. Behavior Research Methods, Instruments, \& Computers, 21, 568-573.

Poizner, H., WoOten, E., \& SALOT, D. (1986). Computergraphic modeling and analysis: A portable system for tracking arm movements in three-dimensional space. Behavior Research Methods, Instruments, \& Computers, 18, 427-433.

Press, W. P., Flannery, B. P., Teukolsky, S. A., \& Vetterling, W. T. (1989). Numerical recipes in Pascal: The art of scientific computing. Cambridge: Cambridge University Press.

Renner, M. J., Pierre, P. J., \& Schilcher, P. J. (1990). Contrast-based digital tracking versus human observers in studies of anımal locomotion. Bulletin of the Psychonomic Society, 28, 77-79.

Scherer, E., MCNicol, R. E., \& CAPEL, M. J. (1992). A computerized system for analyzing linear locomotion. Behavior Research Methods, Instruments, \& Computers, 24, 37-43.

Schmid, G. B. (1991). Chaos theory and schizophrenia: Elementary aspects. Psychopathology, 24, 185-198.

Silva, F. J., \& PEAR, J. J. (1995). Stereotypy of spatial movements during noncontingent and contingent reinforcement. Animal Learning \& Behavior, 23, 245-255.
Silva, F. J., Silva, K. M., \& PEAR, J. J. (1992) Sign- vs. goal-tracking: The effects of conditioned stimulus-to-unconditioned stimulus distance. Journal of the Experimental Analysis of Behavior, 57, 17-31.

Sing, H. C., Thorne, D. R., Hegge, F. W., \& Babkoff, H. (1985). Trend and rhythm analysis of time-series data using complex demodulation. Behavior Research Methods, Instruments, \& Computers, 17, 623-629.

Staddon, J. E. R., \& Simmelhag, V. L. (1971). The "superstition" experiment: A reexamination of its implications for the principles of adaptive behavior. Psychological Review, 78, 3-43.

STURGIS, S. P. (1983). A spectral-analysis tutorial with examples in FORTRAN Behavior Research Methods \& Instrumentation, 15, 377-386.

SySTAT, INC. (1987). Systat: The system for statistics. Evanston, IL: Author.

Timberlake, W., \& Lucas, G. A. (1985). The basis of superstitious behavior Chance contıngency, stimulus substitution, or appetitive behavior? Journal of the Experimental Analysis of Behavior, 44, 279299.

Woltring, X. (1984). On methodology in the study of human movement. In H. T. A. Whiting (Ed.), Human motor actions: Bernstein reassessed (pp. 35-73). Amsterdam: Elsevier, North-Holland.

\section{NOTE}

1. The description of Fourier analysis presented here is summarized from Kaplan's (1983) excellent user-friendly description of Fourier analysis, and we direct those seeking additional information to that article. For mathematıcally onented descriptions of Fourier analysis, see Bloomfield (1976), Brockwell and Davis (1987), and Press et al. (1989).

(Manuscript received May 11, 1994; revision accepted for publication November 23, 1994.) 